\title{
Femtosecond laser pulse written Volume Bragg Gratings
}

\author{
Daniel Richter ${ }^{1 *}$, Christian Voigtländer ${ }^{1}$, Ria G. Krämer ${ }^{1}$, Jens U. \\ Thomas $^{1}$, Andreas Tünnermann ${ }^{1,2}$, and Stefan Nolte ${ }^{1,2}$ \\ ${ }^{1}$ Institute of Applied Physics, Abbe Center of Photonics, Friedrich-Schiller-Universität, \\ Max-Wien-Platz 1, 07743 Jena, Germany \\ ${ }^{2}$ Fraunhofer Institute for Applied Optics and Precision Engineering, Albert-Einstein-Straße 7, \\ 07745 Jena, Germany \\ *Author e-mail address: daniel.richter.1@uni-jena.de
}

\begin{abstract}
Femtosecond laser pulses can be applied for structuring a wide range of transparent materials. Here we want to show how to use this ability to realize Volume-Bragg-Gratings in various - mainly non-photosensitive - glasses. We will further present the characteristics of the realized gratings and a few elected applications that have been realized.
\end{abstract}

OCIS codes: (050.7330) Volume gratings; (050.1950) Diffraction gratings; (140.3390) Laser materials processing

\section{Introcucuon}

Volume Bragg gratings (VBGs) are optical divices possessing a periodic refractive index structure typically inside a glass bulk. Due to their geometrical extension and characteristics they can be employed for a wide range of applications such as narrow spectral or angular filters, (multi-) beam combiners for e.g. fiber or solid state lasers, frequency stabilizers for diode lasers or pulse compressors in case of chirped VBGs. The common fabrication technique is based on exposing photo-thermo-refractive glass to an interference pattern of an UV-laser [1], which, unfortunately, limits the utilized material range to a very small area on the glass map and additionally requires thermal post-processing. Furthermore, the sinusoidal structure of the inscribed grating supports only one spectral component. Making use of femtosecond laser pulses for the inscription of VBGs a wide range of transparent materials, first of all glasses - photosensitve and non-photosensitive ones - can be modified. Here, we want to present our results by employing the phase mask scanning technique [2].

\section{Technique and characteristics}

For our experiments we used a SPECTRA PHYSICS Spitfire system generating down to 50fs pulses at $1 \mathrm{kHz}$ repetition rate with a central wavelength of $800 \mathrm{~nm}$ and a maximum pulse energy of $700 \mu \mathrm{J}$. To generate VBGs we use these pulses and employ the mentioned phase mask scanning technique. Here sample and phase mask are fixed to each other and only the inscribing laser focus is moved. By this one prevents the problem of stitching errors while extending the size of the grating inside the bulk. The needed periodic grating pattern is generated by the \pm 1 . diffraction order of the phase mask. The zeroth diffraction order and thereby the TALBOT-effect can be neglected due to the order walk-off effect. Caused by the lack of stitching errors highly reflective gratings in fused silica could be realized [3].

While employing femtosecond laser pulses the main modification process of the applied material is nonlinear absorption. Hence the induced grating reveals a nonsinusoidal structure which can be decomposed into varios fourier components. Those cause the grating to support several spectral components. Up to now we measured up to the 27 th reflection order of a VBG [4].

While extending the range of applied materials from fused silica to various other we could achieve different homogeneous refractive index modulation contrasts ranging from a few $10^{-4}$ (e.g. Foturan) to several $10^{-3}$ (e.g. fused silica) for different types of glasses. Depending on the refractive index contrast the reflection and deflection behaviour varies, which will be shown and compared with the theoretical expectations and simulations.

\section{Applications}

In the field of laser diode stabilization VBGs are used for generating a narrow spectral feedback. Due to the fixed period of the VBG no additional tuning of the feedback is possible. One chance to overcome this is to make use of the Gouy phase of the free space beam. In the theory on Gaussian beams an additional phase term occurs which pays tribute to the propagation behavior of a Gaussian beam with respect to a plane wave. Thereby the beam adapts a longitudinal phase delay within the Rayleigh length. This so called Gouy phase shift can experimentally be measured when using

This is an Open Access article distributed under the terms of the Creative Commons Attribution License 2.0, which permits unrestricted use, distribution, and reproduction in any medium, provided the original work is properly cited. 


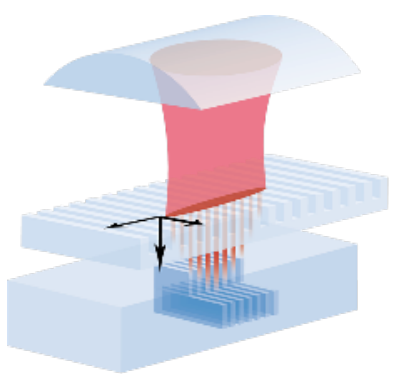

(a) Inscription of a VBG.

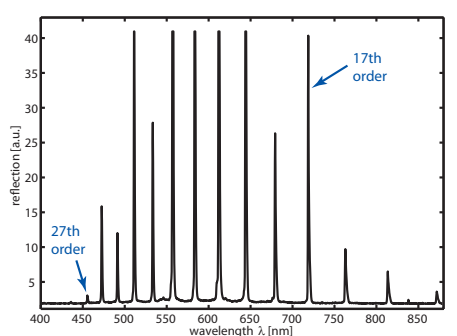

(b) Measurement of higher reflection orders of a VBG with a geometrical period of $\Lambda=4.2 \mu \mathrm{m}$.

Fig. 1: Illustration of the inscription method of a VBG with a phase mask and the spectral behaviour of a VBG with respect to higher reflection orders.

a broadband light emitting fiber and characterizing the reflection behavior of the VBG by adopting the fiber end facet to the grating. Measuring the reflected signal by the help of a circulator while changing the distance between fiber and grating end facet one is able to observe a spectral shift of the central wavelength of the reflected signal which can be attributed to the Gouy phase. The measureable shift is usually in the sub-nm region and can therefore be used for fine-tuning the spectral behaviour of e.g. a stabilized laser diode system.

Another interesting field is the realization of a mulit-beam-combiner. Usually VBGs are known for being spectrally and angularly selective elements that can be used for beam combining - but one element is required for each wavelength. To overcome this limitation one has to shift to two- or three-dimensional structured VBGs. Those work similar to the 'von Laue' experiment for investigating the structure of crystals [5, 6] and support a discrete set of spots, each of them well defined at wavelength and direction. The main difference is the geometrical paramters of the unit cell which shifts the wavelength of the supported discrete diffraction spots from the x-ray region to the UV-VIS-IR range. Here, we want to show first results of our two-dimensional prototype. Furthermore, this technique can be used to learn more about crystal geometries by adapting and realizing their parameters and investigating the discrete diffraction pattern with this model system.

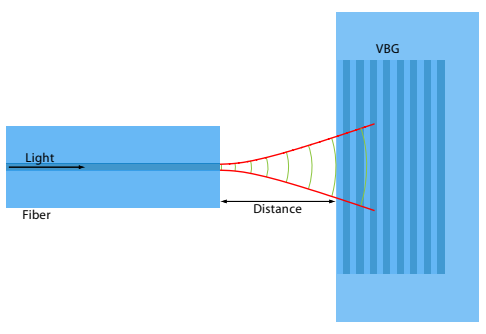

(a) Schematic setup for measuring the spectral Gouy-shift based on the narrow reflection bandwidth of a VBG.

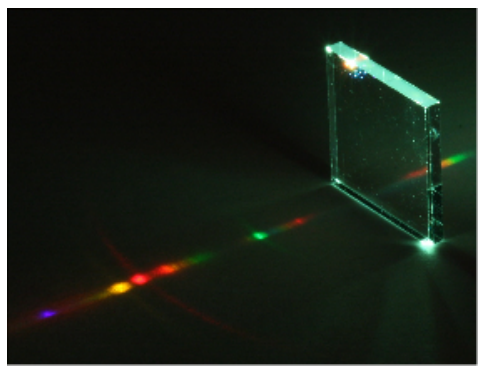

(b) Discrete diffraction spots of a twodimensional VBG while illuminated with a supercontinuum source.

Fig. 2: Two examples for the application of fs pulse written VBGs.

\section{References}

1. O. M. Efimov, L. B. Glebov, L. N. Glebova, K. C. Richardson, and V. I. Smirnov, "High-efficiency bragg gratings in photothermorefractive glass," Appl. Opt. 38, 619-627 (1999).

2. J. Thomas, E. Wikszak, T. Clausnitzer, U. Fuchs, U. Zeitner, S. Nolte, and A. Tünnermann, "Inscription of fiber bragg gratings with femtosecond pulses using a phase mask scanning technique," Applied Physics A 86, 153-157 (2007).

3. C. Voigtländer, D. Richter, J. U. Thomas, A. Tünnermann, and S. Nolte, "Inscription of high contrast volume bragg gratings in fused silica with femtosecond laser pulses," Appl. Phys. A: Mater. 102, 35-38 (2011).

4. D. Richter, C. Voigtländer, R. G. Becker, J. U. Thomas, A. Tünnermann, and S. Nolte, "Efficient volume bragg gratings in various transparent materials induced by femtosecond laser pulses," in "CLEO/Europe and EQEC 2011 Conference Digest," (2011).

5. M. von Laue, "Zur optik der raumgitter," Physikalische Zeitschrift 14, 1286 (1913).

6. P. P. Ewald, "Introduction to the dynamical theory of x-ray diffraction," Acta Crystallogr. A 25, 103-108 (1969). 